\title{
Luminosity-Distances of IUE observed Active Galaxies
}

\section{Vijayakumar H Doddamani and P. Vedavathi}

Dept. of Physics, Bangalore University, Bangalore-560056

email: drvkdmani@gmail.com

\begin{abstract}
Active galaxies are the most luminous objects observed in the Universe and are believed to be powered by mass accretion processes taking place in the vicinity of the central Super massive black hole $\left(\mathrm{M}_{B H} \geqslant 10^{8} \mathrm{M}_{\text {sun }}\right)$. However, the details of the power generation mechanisms are not understood well yet. In this paper, we are presenting a comparative study of luminositydistance estimations for the complete sample of active galaxies observed by IUE satellite by different methods. IUE has made UV spectroscopic observations of nearly 400 active galaxies comprising mostly Seyfert 1 galaxies and quasars. We have chosen all the active galaxies observed by IUE satellite for the study of luminosity-distance with redshift. The luminosity-distances $\left(\mathrm{D}_{L}\right)$ have been calculated using the Hubbles law under non-relativistic and relativistic limits with $\mathrm{H}_{0}=73 \mathrm{Km} / \mathrm{sec} / \mathrm{Mpc}$ and Terrell (1979) also. We have found that all $\mathrm{D}_{L}$ estimations are consistent with each other for $\mathrm{z} \leqslant 1$ and diverge for $\mathrm{z} \geqslant 1$. The results of cosmological calulator I and II are found to consistent with each other and higher by several factors over cosmological calculator IV and the predictions of the Hubble's law under relativistic case. We observe a kind bimodal distributions in $\mathrm{D}_{L}$ for $\mathrm{z} \leqslant 3.5$.
\end{abstract}

Keywords. Active galaxies, Seyfert 1 galaxies, quasars, low redshift, Luminosity-distance

\section{Introduction}

The active galaxies are the most luminous objects observed in the Universe and are believed to be powered by mass accretion processes taking place in the vicinity of the central super massive black hole $\left(\mathrm{M}_{B H} \geqslant 10^{8} \mathrm{M}_{\text {sun }}\right)$. Thus, it becomes very much important to understand the issue of their luminosity-distance $\left(\mathrm{D}_{L}\right)$ estimations. There are no direct methods available for the distance estimation of active galaxies independent of their redshifts. The redshift-independent distance estimation methods are reliable only for the galactic scale. Hubbles law cannot be used in its simple form to estimate $\mathrm{D}_{L}$ estimations at higher redshift active galaxies. Active galaxies serve as standard-distance candles for the distance estimations on cosmological scales. To look into the consistent $\mathrm{D}_{L}$ estimation issues of active galaxies especially for $\mathrm{z} \geqslant 1$, we have undertaken the $\mathrm{D}_{L}$ estimations of the complete sample of IUE observed active galaxies using simple Hubbles law under both non-relativistic and relativistic limits (Bogess et al. 1978). We have made use of the following relations for $\mathrm{D}_{L}$ estimations:

$$
\begin{gathered}
D_{L}=c z / H_{0} \\
D_{L}=V_{R} z / H_{0} \\
(1+z)^{2}=\left(c+V_{R}\right) /\left(c-V_{R}\right) \\
V_{R}=K c
\end{gathered}
$$

where K- a numerical constant and c-velocity of light. These estimations are compared with the results of $\mathrm{D}_{L}$ estimations using Terrell (1979) formula and cosmological 


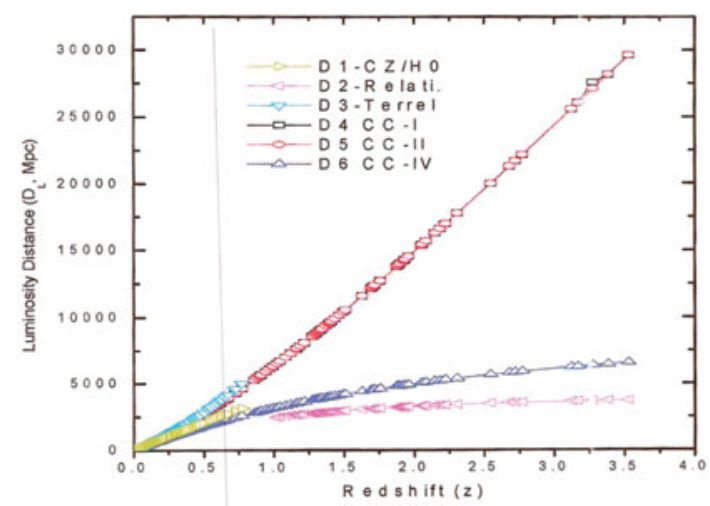

Figure 1. Dependence of Luminosity-Distances (DL, Mpc) with redshift (z) for IUE observed active galaxies.

calculators available online at http://ned.ipac.caltech.edu. A cosmological model of $\omega=0.7$ and $\omega_{m}=0.3$ with $\mathrm{H}_{0}=73 \mathrm{Km} / \mathrm{sec} / \mathrm{Mpc}$ has been assumed for all the calculations.

\section{IUE Database}

The IUE satellite has made low resolution UV spectroscopic observations of Seyfert galaxies (Sy1 and Sy2), Quasars, BL Lac HP objects, Radio galaxies and HII galaxies in the 1200-3000 $\AA$ wavelength range (Bogess A. et al. 1978). In order to study the line luminosity dependence with the UV continuum, we have undertaken luminosity-distance $D_{L}$ calculations for all the 412 active galaxies for their redshift $\mathrm{z} \leqslant 3.5$.

\section{Conclusions and Discussions}

We have found that all $D_{L}$ estimations are consistent with each other for $\mathrm{z} \leqslant 0.6$ from and diverge for $z \geqslant 0.6$. The results of cosmological calculator I and II $\left(D_{4}\right.$ and $D_{5}$ in fig. 1) are found to consistent for all values of $\mathrm{z}$ with each other. The $D_{L}$ estimations from Hubbles law $\left(\mathrm{D}_{1}\right)$ under non-relativistic limit, cosmological calculator IV $\left(\mathrm{D}_{6}\right)$ and relativistic Hubble law $\left(\mathrm{D}_{2}\right)$ have all yielded lower values by higher by several factors over cosmological calculator I and II. Thus, we have found a kind of bimodal distributions in $D_{L}$ for $\mathrm{z} \leqslant 3.5$. The results imply that the $D_{L}$ estimations methods are not able to produce unique distance value for an object in higher redshift regime $(z \geqslant 3.5)$.

\section{Acknowledgements}

This work has been carried out under a UGC major research project sanctioned to the first author. We also acknowledge financial support of UGC, New Delhi, and Bangalore University with gratitude towards carry out this research work.

\section{References}

Bogess, A., et al. 1978, Nature 275, 372 \& 377

Peterson, B. M. 1997, An Introduction to Active Galactic Nuclei (CUP)

Schmidt, B. \& Riess, A. 1998, AJ 116, 1009

Cappi, A. 2001, ApLEC 40, 161

Perlmutter, S., et al. 1999, ApJ 517, 565

Perlmutter, S., et al. 1995, ApJ 440, L41

Terrell, J. 1977, A. J. P. 45, 869

Wright, E. L. 2006, PASP 118, 1

Wright, E. L. 2006, PASP 118, 1711

Doddamani, V. H. 2004, Ultra Violet Spectroscopic study of Seyfert 1 galaxies and quasars

(Thesis submitted to Bangalore University), Bangalore, India 